\title{
Correction: Symptom improvement in children with autism spectrum disorder following bumetanide administration is associated with decreased GABA/glutamate ratios
}

Lingli Zhang, Chu-Chung Huang, Yuan Dai, Qiang Luo, Yiting Ji, Kai Wang, Shining Deng, Juehua Yu, Mingyu Xu, Xiujuan Du, Yun Tang, Chun Shen, Jianfeng Feng, Barbara J Sahakian, Ching-Po Lin (1) and Fei Li

\section{Correction to: Translational Psychiatry} https://doi.org/10.1038/s41398-020-0692-2 published online 27 January 2020

An important detail was omitted in the Method of the original Article, I.E, The CARS and other evaluations were conducted 'blind' to condition (Bumetanide or no treatment) by experienced clinicians. This has now been updated in the HTML and PDF versions of this Article.

Published online: 12 February 2020 